\title{
Ultralow-threshold microcavity Raman laser on a microelectronic chip
}

\author{
T. J. Kippenberg, S. M. Spillane, D. K. Armani, and K. J. Vahala \\ California Institute of Technology, Department of Applied Physics, Pasadena, California 91125
}

Received November 25, 2003

\begin{abstract}
Using ultrahigh- $Q$ toroid microcavities on a chip, we demonstrate a monolithic microcavity Raman laser. Cavity photon lifetimes in excess of $100 \mathrm{~ns}$ combined with mode volumes typically of less than $1000(\mu \mathrm{m})^{3}$ significantly reduce the threshold for stimulated Raman scattering. In conjunction with the high ideality of a tapered optical fiber coupling junction, stimulated Raman lasing is observed at an ultralow threshold (as low as $74 \mu \mathrm{W}$ of fiber-launched power at $1550 \mathrm{~nm}$ ) with high efficiency (up to $45 \%$ at the critical coupling point) in good agreement with theoretical modeling. Equally important, the wafer-scale nature of these devices should permit integration with other photonic, mechanical, or electrical functionality on a chip. (C) 2004 Optical Society of America
\end{abstract}

OCIS codes: $190.5650,230.5750,220.4000$.

Stimulated Raman scattering in silica allows generation of light and conversion of light to new frequencies and can, because of the large transparency window of silica, provide broadband optical gain. Stimulated Raman scattering can also be used to extend the wavelength range of state-of-the-art semiconductor sources into new wavelength bands and as such is important in sensing, spectroscopy, and photonics. However, because of the necessity of high field intensity and (or) long interaction length, Raman lasers generally require high pump powers and tabletop dimensions. Ultrahigh- $Q$ microcavities allow a significant reduction of the necessary pump power, ${ }^{1-4}$ and progress toward miniaturization of this class of devices has been made with the demonstration of a microcavity Raman laser that uses a tapered-fiber waveguide coupled to an ultrahigh- $Q$ microsphere. ${ }^{1}$ However, microsphere properties such as size and shape are not easily controlled during fabrication, nor are microspheres readily integrable with other optical devices or electronics. In this Letter we present what is to our knowledge the first demonstration of a chip-based (monolithic) microcavity Raman laser, using recently developed ultrahigh- $Q$ toroid microcavities. ${ }^{5}$ We show that these devices are excellent candidates for nonlinear oscillation based on Raman gain, as they exhibit microwatt-level stimulated Raman thresholds and high pump-to-Raman conversion efficiencies (up to $45 \%$ has been observed). In comparison with Raman oscillation in microspheres, ${ }^{1}$ these devices oscillate in single mode over a wide range of pump powers and exhibit lower effective Raman oscillation thresholds because of their reduced mode volume with respect to a spherical microcavity.

The fabrication of wafer-based toroid microcavities uses a combination of conventional silicon microfabrication techniques and a $\mathrm{CO}_{2}$-laser-assisted reflow process and is described in detail in Ref. 5. The reflow process creates an exceptionally smooth dielectric interface and makes possible ultrahigh $Q$ factors in excess of $100 \times 10^{6}$. Figure 1 shows a scanning electron micrograph of a toroid microcavity.
To excite microtoroid whispering-gallery modes (WGMs) efficiently, tapered optical fiber waveguides were used. ${ }^{6}$ These waveguides were fabricated by heating and adiabatically stretching an optical fiber. The tapers used in the present study typically had waist diameters of $1-2 \mu \mathrm{m}$, insertion loss of less than $5 \%$, and a flat transmission spectrum $(<5 \%$ modulation from unity transmission). Evanescent coupling was achieved by bringing the tapered optical fiber in close proximity with the periphery of a toroid microcavity. The coupling behavior of the waveguideresonator system can be modeled with the intrinsic photon lifetime $\left(\tau_{0}\right)$ and the external (coupling-related) lifetime $\left(\tau_{\text {ex }}\right)$. The coupling regime is described by the dimensionless coupling parameter $K \equiv \tau_{0} / \tau_{\mathrm{ex}}$, where, following standard conventions, ${ }^{7} K<1$ designates undercoupled; $K=1$, critically coupled 6 ; and $K>1$, overcoupled. Changing the taper-microtoroid separation permits control of the coupling parameter. The toroidal WGMs were pumped with a narrow-linewidth

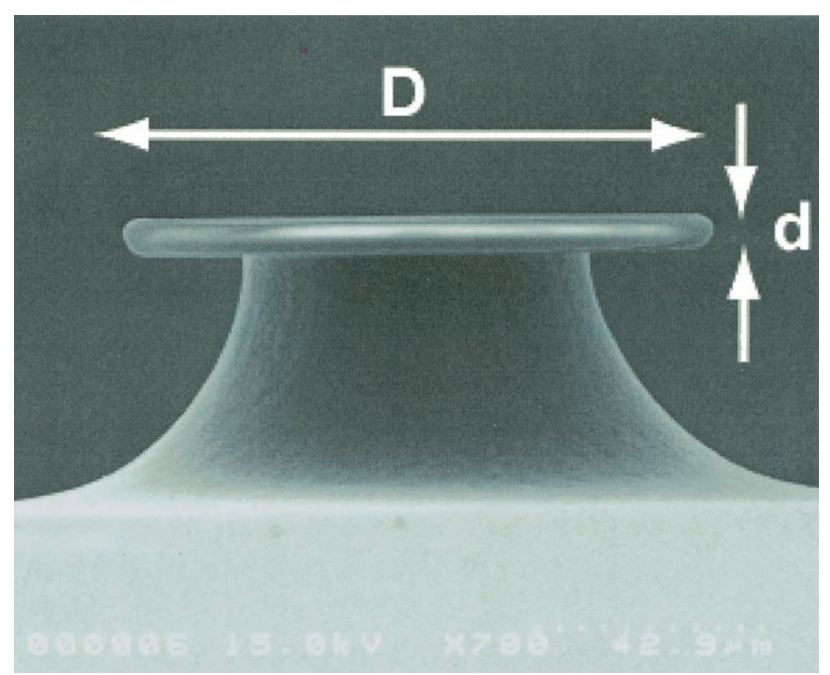

Fig. 1. Scanning electron microscope side profile of a toroid microcavity showing the principal $(D)$ and minor $(d)$ toroid diameters. 
$(<300-\mathrm{kHz})$ external-cavity tunable laser operating in the 1550-nm telecommunication band. We achieved phase matching to the pump mode (and Raman mode) by varying the taper waist in the coupling region. In this way high ideality could be achieved for coupling to the WGM modes. ${ }^{8}$ Both codirectional and contradirectional coupling of the cavity Raman mode into the tapered optical fiber were measured with dual optical spectrum analyzers.

Figure 2 shows a typical Raman emission spectrum for a critically coupled microtoroid WGM pumped above Raman threshold. The pump wavelength is located at $1550 \mathrm{~nm}$, and the Raman emission is present in the 1650-nm band, downshifted by approximately $12.5 \mathrm{THz}$ relative to the pump mode. It is significant to note that the emission was observed to be single mode. Single-mode operation was generally observed over a large range of pump powers and, in contrast to microspheres, is a result of the strongly reduced azimuthal degrees of freedom within the toroidal cavity. This single-mode behavior constitutes a significant improvement with respect to prior work on microspheres ${ }^{1}$ or microdroplets, ${ }^{3,4}$ which exhibit multimode oscillation spectra because of the presence of numerous nearly degenerate azimuthal modes.

The dependence of the Raman emission as a function of pump power for a critically coupled microtoroid is given in the inset of Fig. 2 and displays clear threshold behavior. The maximum Raman output power for this microtoroid was more than $150 \mu \mathrm{W}$ (bidirectional), and the measured pump-to-Raman differential conversion efficiency was $45 \%$. The latter number is close to the theoretically expected launched pump-to-Raman differential conversion efficiency in the absence of any parasitic losses, which is $\sim 47 \%$ at the critical point, as given by

$$
\frac{\mathrm{d} P_{\text {Raman }}}{\mathrm{d} P_{\text {pump }}}=\eta_{\mathrm{ex}}=2 \frac{\lambda_{p}}{\lambda_{R}}\left(1+\frac{1}{K}\right)^{-2} .
$$

Analysis of stimulated Raman scattering in a microcavity, under the assumption of an ideal coupling junction, ${ }^{8}$ yields the threshold equation ${ }^{1}$

$$
\begin{aligned}
P_{\text {thresh }}= & C(\Gamma) \frac{\pi^{2} n^{2}}{\lambda_{p} \lambda_{R} g_{R}} V_{\text {eff }}\left(\frac{1}{Q_{0}}\right)_{P}\left(\frac{1}{Q_{0}}\right)_{R} \\
& \times \frac{\left(1+K_{p}\right)^{2}}{K_{p}}\left(1+K_{R}\right) .
\end{aligned}
$$

In Eq. (2), $n$ is the index of refraction (silica $n=1.445), \lambda_{R}$ and $\lambda_{p}$ are the wavelengths of the Raman and the pump modes, $V_{\text {eff }}$ is the effective mode volume (which is equal to twice the mode volume), $K_{p}$ and $K_{R}$ describe the coupling parameters of the pump and the Raman modes, and $Q_{0}$ designates the intrinsic quality factor. The Raman gain coefficient (silica $0.66 \times 10^{-13} \mathrm{~m} / \mathrm{W}$ at $1.55 \mu \mathrm{m})$ is given by $g_{R}$. Parameter $C(\Gamma)$ takes a value between 1 and 2 and describes the reduction of circulating power caused by excitation of the counterpropagating WGM. ${ }^{9}$ Equation (2) scales as $\propto 1 / Q^{2}$, reflecting the fact that a high $Q$ factor creates a twofold benefit of reduced round-trip loss and increased Raman gain.
In addition, the Raman threshold scales linearly with effective mode volume $V_{\text {eff }}$. Since the mode volume of the toroidal WGMs is expected to be reduced as the minor diameter (see Fig. 1) is reduced for fixed $D$, lower-threshold operation is expected in a microtoroid than in the case of a microsphere that has diameter $D$ and a comparable $Q$. Conversely, measurement of the Raman threshold allows the mode volume to be inferred by inversion of Eq. (2). We use this method below to infer the toroid mode volume with varying minor diameter as well as to compare the mode volume of toroids and spheres with equivalent principal diameters. For this study toroid microcavities were fabricated with an approximately constant principal diameter $(D)$ of $55 \mu \mathrm{m}$ and minor diameters $(d)$ varying from 3.7 to $\sim 10 \mu \mathrm{m}$. The quality factors were measured by cavity ringdown, as used in the study reported in Ref. 6, and had values near $100 \times 10^{6}\left(\tau_{0} \sim 80 \mathrm{~ns}\right)$. The observed Raman thresholds varied in the range 74-250 $\mu \mathrm{W}$. To compare the threshold among different cavity geometries, we normalized the threshold formula [Eq. (2)] to take into account the different resonator characteristics, leaving only the modal volume dependence. The experimental protocol proceeded by determining the loaded $Q$ factor and modal coupling parameter $\Gamma .^{9}$ To correct for variation of Raman gain coefficient $g\left(\lambda_{R}, \lambda_{p}\right)$, both pump wavelength $\lambda_{p}$ and Raman emission wavelength $\lambda_{R}$ were also measured. Subsequently, scanning electron microscopy was used to record the side profiles of the microcavities to determine the effective toroid minor diameter $(d)$. The mode volume was then retrieved from the minimum Raman threshold measurement by inversion of Eq. (2). Figure 3 presents the experimental results of this procedure and plots the effective mode volume of the toroid inferred from

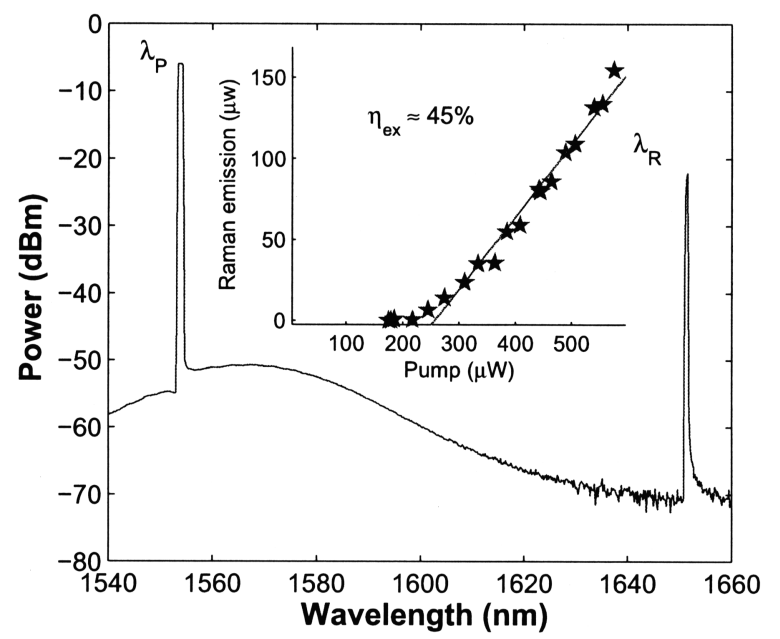

Fig. 2. Raman emission spectrum of a toroid microcavity showing single-mode oscillation. The pump is located at $1550 \mathrm{~nm}$, and the Raman emission is shifted $12.5 \mathrm{THz}$ into the 1650-nm band. Inset, bidirectional Raman emission as a function of pump power for a 58- $\mu \mathrm{m}$-diameter toroid microcavity $\left(Q_{0}=0.6 \times 10^{8}\right)$ at the critical point. The threshold is $\sim 250 \mu \mathrm{W}$, and the bidirectional conversion efficiency is $\sim 45 \%$. 


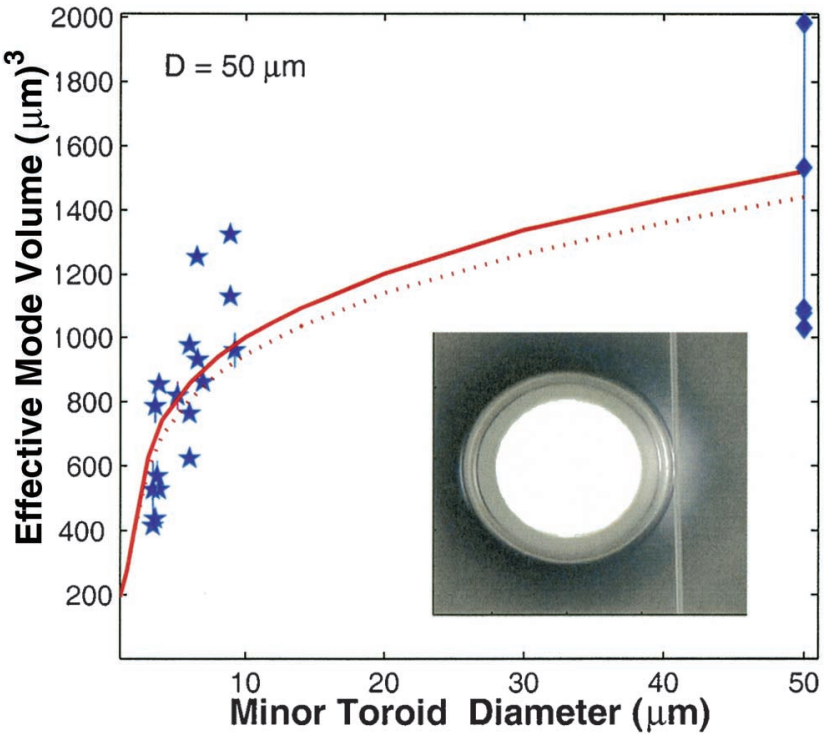

Fig. 3. Main figure: experimental and theoretical effective mode volume ${ }^{10}$ (in cubic micrometers) of toroid microcavities as a function of the toroid minor diameter $(d)$ for fixed principal diameter $D(D=50 \mu \mathrm{m})$. The case $d=D$ corresponds to a microsphere, and the data shown are taken from Ref. 1 for comparison. The solid and dotted curves refer to the mode volume of a fundamental toroidal WGM (TM and TE case, respectively) obtained by numerical modeling. Inset, optical micrograph of an $\sim 80$ - $\mu \mathrm{m}$-diameter toroid microcavity coupled to a tapered optical fiber.

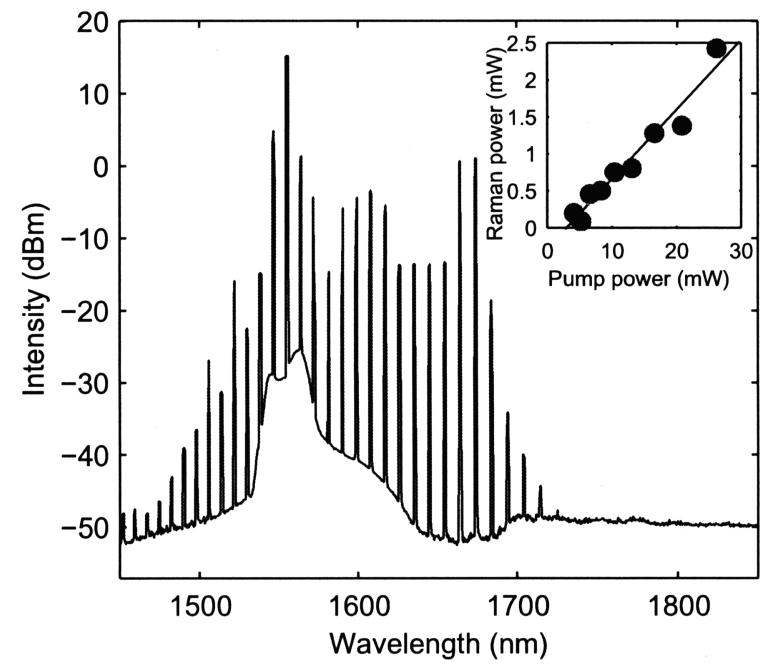

Fig. 4. Broadband frequency generation in a microtoroid Raman laser pumped far above threshold at 1550-nm wavelength. Inset, $2.5 \mathrm{~mW}$ of output power from a single Raman emission wavelength near $1680 \mathrm{~nm}$. The modes are spaced with the free spectral range of the cavity.

the measurement process. For comparison, the solid (dashed) curve is the theoretically expected volume dependence based on a calculation of the TM (TE) fundamental mode volume for a 50 - $\mu$ m-diameter microtoroid. Despite experimental uncertainty that was due to the inability to measure photon life-times in the $1600-\mathrm{nm}$ band, the measured volume depen- dence is in reasonable agreement with the calculation. The lowest observed threshold in this measurement occurred for the smallest toroidal minor diameter $\left(D \approx 61 \mu \mathrm{m}, d \approx 3.7 \mu \mathrm{m}\right.$, and $\Gamma \approx 1.3, Q_{0}=1.0 \times 10^{8}$, with $P \approx 74 \mu \mathrm{W}$, in agreement with the Raman threshold formula). Also, for comparison purposes, data obtained from a previous study ${ }^{1}$ that used silica microspheres with a comparable diameter $(d=D)$ are included in Fig. 3.

Nonlinear processes in silica microtoroids were also observed when the toroid microcavities were pumped at high power levels. By use of an erbium-doped fiber amplifier to boost the external-cavity pump wave to power levels of $\sim 25 \mathrm{~mW}$, a highly nonlinear emission spectrum is produced with a $53-\mu \mathrm{m}$-diameter microtoroid (Fig. 4). The spectrum consists of frequency redshifted stimulated Raman modes as well as blueshifted modes that are due to Raman-assisted four-wave mixing. It is important to note that all the participating modes in this nonlinear process are single azimuthal modes, spaced by the free spectral range of the cavity $(10 \mathrm{~nm})$. The generated radiation spans nearly $35 \mathrm{THz}$, and the highest fiber-coupled power of individually oscillating modes is nearly $2.5 \mathrm{~mW}$ (see the inset of Fig. 4).

In summary, we have demonstrated what is to our knowledge the first Raman laser on a chip. The Raman microlasers exhibit ultralow threshold, high efficiency, and single-mode emission. Also, they are coupled with high ideality by use of tapered optical fibers. The lasers are fabricated with standard silicon processing techniques, allowing integration of Raman microlasers with complementary optical, mechanical, or electrical functionality.

This work was funded by the Defense Advanced Research Projects Agency and the National Science Foundation. K. J. Vahala's e-mail address is vahala@ caltech.edu.

\section{References}

1. S. M. Spillane, T. J. Kippenberg, and K. J. Vahala, Nature 415, 621 (2002).

2. V. B. Braginsky, M. L. Gorodetsky, and V. S. Ilchenko, Phys. Lett. A 137, 393 (1989).

3. R. K. Chang and A. J. Campillo, Optical Processes in Microcavities, Vol. 3 of Advanced Series in Applied Physics (World Scientific, Singapore, 1996).

4. H. B. Lin and A. J. Campillo, Phys. Rev. Lett. 73, 2440 (1994).

5. D. K. Armani, T. J. Kippenberg, S. M. Spillane, and K. J. Vahala, Nature 421, 925 (2003).

6. M. Cai, O. Painter, and K. J. Vahala, Phys. Rev. Lett. 85, 74 (2000).

7. H. A. Haus, Electromagnetic Fields and Energy (Prentice-Hall, Englewood Cliffs, N.J., 1989).

8. S. M. Spillane, T. J. Kippenberg, O. J. Painter, and K. J. Vahala, Phys. Rev. Lett. 91, 043902 (2003).

9. T. J. Kippenberg, S. M. Spillane, and K. J. Vahala, Opt. Lett. 27, 1669 (2002).

10. $V_{\text {eff }} \equiv\left(\int\left|\vec{E}_{p}\right|^{2} \mathrm{~d} V \int\left|\vec{E}_{R}\right|^{2} \mathrm{~d} V\right) / \int\left|\vec{E}_{p}\right|^{2}\left|\vec{E}_{R}\right|^{2} \mathrm{~d} V$ and is approximately twice the mode volume. 